\title{
Web-based Kanban Tool for Project Management
}

\author{
Lawrence Toh Yee Sheng ${ }^{1}$, Sivaguru A/L Subarmaniyan ${ }^{2, *}$ Nur Amira Abdul Majid ${ }^{3}$ \\ ${ }^{1,2,3}$ School of Computing, Asia Pacific University of Technology \& Innovation, Malaysia \\ "Corresponding author.Email: sivaguru@staffemail.apu.edu.my
}

\begin{abstract}
The purpose of this project is to develop a web-based application that assist the end users in managing their projects. With the Kanban concept as its core, the system will provide the benefits of using a traditional Kanban board to the users through the web. This project is to provide a web-based project management solution to the users. Through the first leg of the project, the researcher found out that the target audience, students did poorly in managing their assignments and projects, resulting in problems such as late submission. Thus, a solution is required to solve this issue, and the idea of a Web-based Kanban Tool is proposed.
\end{abstract}

Keywords: Project management, Web development, Kanban.

\section{INTRODUCTION}

A life cycle of a project is divided into 5 different processes which are initiating, planning, executing, controlling, and closing [1]. These 5 processes are large group of processes that must take place within a project life cycle and are interrelated to each other. Thus, as a team working on a project, the team requires a way to manage the three building blocks of a project, time, budget, and scope, monitor the progress of the project, an agreed direction regarding the project's goal and a way to maintain communications with stakeholders [2] [10-16]. This is where project management comes in. Project management is an important skillset where an individual applies their knowledge, skills, tools, and techniques onto their project, so that project requirements could be fulfilled [3]. Conducting a project without project management is a fool's errand which will cause mismanagement of budget, confusion, miscommunication, clashes, nothing to hold the team and client together and finally lead to the failure of the project [4] [17-23].

The action of conducting a project without project management is much more noticeable within the learning environment as late submission of the projects or assignments are a common occurrence. Thus, as a solution, this report proposes a web-based Kanban tool to encourage and motivate students to have a habit of conducting project management whenever handling a project or assignment.
Through the implementation of the proposed system, significant improvement regarding how students handle their projects or assignments could be identified as a viable way to conduct project management would be provided.

\section{MATERIALS AND METHODS}

\subsection{Front End Development}

The programming languages that will be used for front end development will be the three essential technologies of web content production, Hypertext Markup Language (HTML), Cascading Style Sheets (CSS) and JavaScript (JS).

HTML is the most basic building block of the web as it is the standard markup language that is used for creating web pages and it defines the structure of a web content [5]. CSS on the other hand is a style sheet language which is recommended programming language to be learned after HTML as it is used to style and lay out the elements of a web page which is written in HTML [6]. Following that, JS is a lightweight programming language that enables interactivity within a web page [7]. While HTML provide structure and CSS provide style to a web page, JS provide interactive elements to a web page. 


\subsection{Back End Development}

The programming language which will be used for the back-end development will be JavaScript (JS). The main reason why JavaScript is chosen as the programming language for developing the back-end is to simplify the development process. Since JavaScript is one of the essential programming language of web content production, it is very likely that the developer is already familiar with it. Thus, eliminating the requirement of learning another programming such as $\mathrm{C \#}$ or Python, it makes the development process for the back-end much more straight forward.

\subsection{Back End Development}

One of the frameworks that would be implemented in the front-end development of this project would be Vue.js. Created by Evan You in 2014, Vue.js is a progressive and incrementally adoptable JavaScript framework which is used for building user interfaces on the web [8]. The advantages of Vue.js lies in its flexibility and the ease of use. Furthermore, the simple structure of Vue.js makes it easy to be learned and understood. Moreover, Vue.js is known for its flexibility as it enables developers to write the template in either HTML file, JS file and pure JS file using virtual nodes [8].

Alongside Vue.js, Vuetify would also be implemented in the front-end development of this project. Vuetify is a material design component framework that is built on top of Vue.js [9]. This means that the combination of Vue.js and Vuetify makes it much easier to build and style web pages which are rich and provides a more engaging user experience.

The last framework that would be implemented in this project would be Feathers JS. Feathers JS would be used in developing the back-end of this project. Feathers is a lightweight web-framework which is built on top of the Express framework for creating RESTful APIs and realtime applications using the Socket IO library. Besides that, Feathers JS also contains the ability to interact with multiple front-end technology such as React, Angular and most importantly Vue.js through Feathers Vuex, thus making it a great choice as a back-end web framework for this project.

\section{OVERVIEW OF WEB-BASED KANBAN TOOL}

Kanban system is generally a simple and an effective tool used to perform the pull concept of lean manufacturing and this Kanban system is considered as essential component of the Toyota production system. If the demand is frqeuenct and stable, this Kanban system effectively works at the shop floor control. Moreover, it is also applied to the customers, suppliers and also in global supply chain for simplifying the Just-In-Time (JIT) delivery.

\subsection{System configuration}

The Web-based Kanban tool is required to be configured for virtually map the actual manufacturing system's procedures. The configuration interface of system is used for defining the tool configurations.

The settings and parameters required to configure the Kanban tool are given as follows:

1. Materials data: It comprises the information about the start-up inventory level, material names, descriptions, etc.

2. Operation's data: It includes the performance standards, work instructions, essential quantity, standard operations procedures and so on.

3. Connection between operations and materials: For creating the bill of materials.

4. Connection among the workstations and operations: For choosing the available workstations and generate the routing of materials.

5. Size of buffer: For restricting the inventory level and Work-In-Process (WIP).

6. Reordering point: to obtain material from upstream processes.

7. User groups: For individual users, a suitable tasks and functions are assigned and displayed during $\log$ in.

The actual manufacturing system and updated response are used to configure the Web-based system using system administrator. The text-based input boxes shown in the Figure 1 is used by the system configuration interface for selecting/ entering the required data. The virtual system's configuration is reviewed by the system administrator, once an all data is precisely configured in the interface. A vertical value stream map is utilized for the graphical demonstration of each product type or material. The developed map integrates the bill of material as shown in Figure 2. The tasks are performed by the workstations and therefore, the material routing is graphically shown in Kanban tool. Additionally, each material's level of WIP is illustrated for the monitoring purpose. 


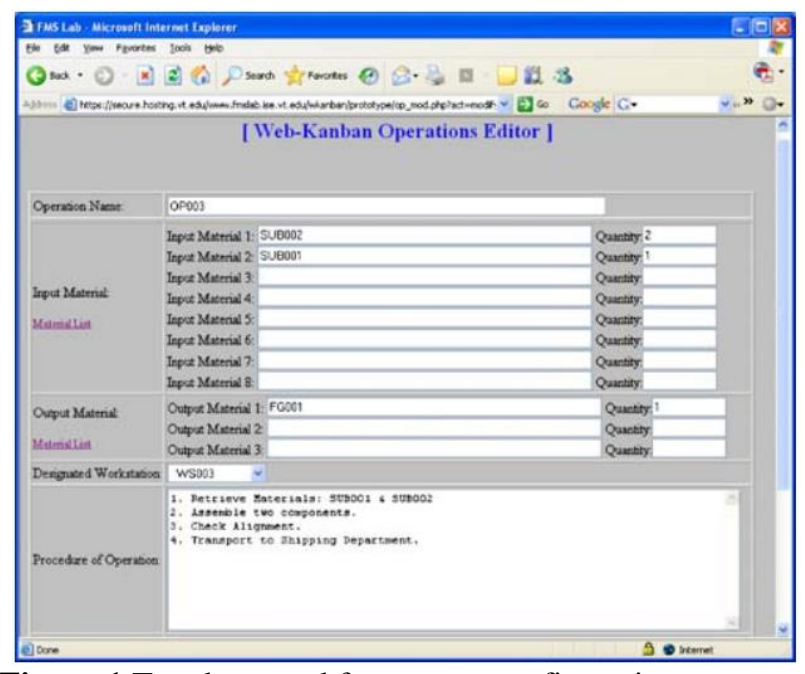

Figure 1 Text box used for system configuration

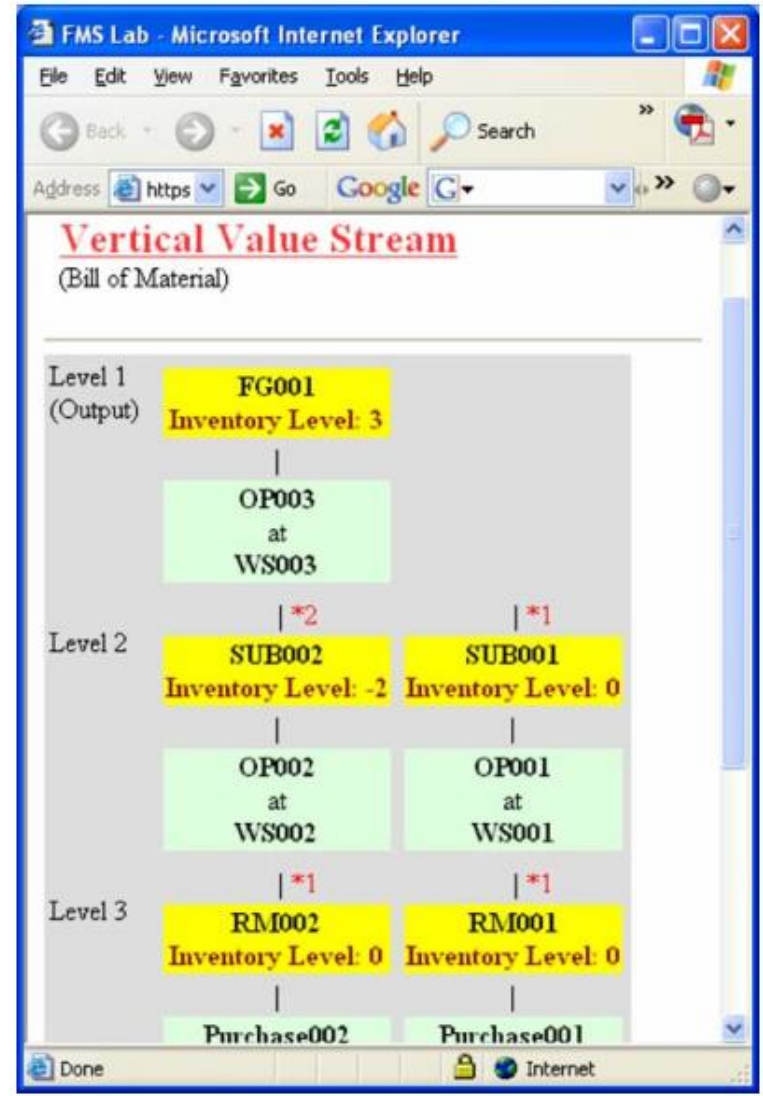

Figure 2 Vertical value stream map

\subsection{Working process of Web based Kanban operations}

In the Kanban system, the jobs are initialized when the Kanban is acquired from the management unit or from the downstream workstation. The pull production system initialized by the Web-based Kanban is shown in the Figure 3. The management unit or downstream workstation transmits the pull signals (Kanbans) for each workstation.
The selected tasks are accomplished by the workstation and the information about the performances are updated to the server. After completing the tasks, the products are reallocated to the online buffers, inventory or to the successive workstation based on the requirement.

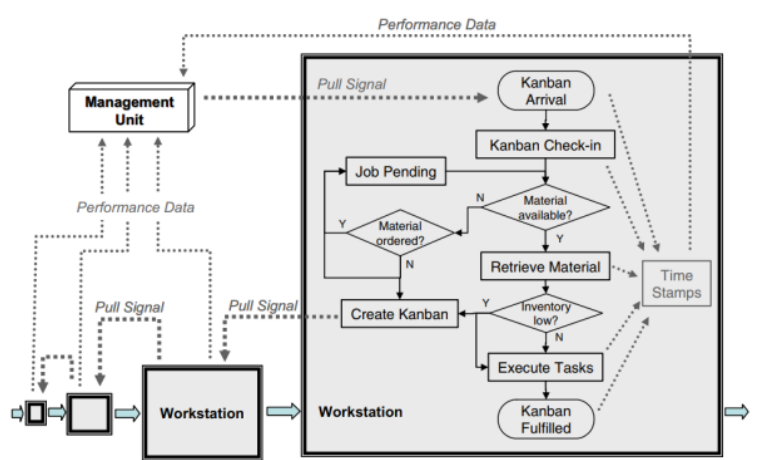

Figure 3 Workflow of Web based Kanban tool

\section{RESULTS AND DISCUSSION}

After analyzing the results gathered through the data collection method conducted by the researcher, the researcher can conclude that project management methodology is important to be implemented when conducting assignments. Some major factors that lead to this conclusion are the struggles that the respondents faced during their assignments. This conclusion is also backed up by the response from the respondents as all the respondents agree that project management methodology should be implemented in their assignments. Besides that, the researcher is also able to conclude that the elements of Kanban are vital in improving the quality of work as the respondents agree that the visualization of tasks can assist them in monitoring the progress of their project/assignment, the characteristic of adding a work limit helps to improve productivity and the importance of prioritizing tasks.

Moving on to the development and testing phase, the user acceptance test is performed to see if the developed system meets the requirements. After analyzing the feedbacks, it could be concluded that the develop system meets its requirement of providing a web-based project management solution to the users.

\section{CONCLUSIONS}

The purpose of this project is to provide a web-based project management solution to the users. Through the first leg of the project, the researcher found out that the target audience, students did poorly in managing their assignments and projects, resulting in problems such as late submission. Thus, a solution is required to solve this issue, and the idea of a Web-based Kanban Tool is proposed. 
Through the development and testing of the proposed system, it could be concluded that the end users is able to obtain multiple beneficial results by using the proposed system. Using the Kanban concept as the core idea of this system, the system is developed with the idea to provide the benefits of using a traditional Kanban method to the users. Thus, by using the system, it helps the user in improving the delivery of their products by eliminating bottlenecks, improve flow and reduce cycle time. As a web application, the system is also highly accessible for the users as they will be able to access the system easily through the internet.

Most importantly, as a collaborative tool, the proposed system enables team collaboration as users can collaborate with other users in managing their project using a single Kanban board. By doing so, it makes group projects much more efficient by assisting team members to have a better grasp on the progress and situation of their project. Moreover, the proposed system will also help the team to be more responsive through the continuous delivery of endproduct and faster gathering of feedbacks.

Overall, it could be can agreed that the developed system, Web-based Kanban Tool meets the user requirements and objectives by achieving what it promised to deliver.

\section{ACKNOWLEDGMENTS}

First and foremost, I would like to express my gratification to the respondents of my questionnaire. By using their time to complete the questionnaire with truthful and genuine response, it enables me to gain a better understanding regarding the topic which is being researched. It has also given me the opportunity to understand the respondents' perspective regarding the topic at hand. Overall, the participation of the people mentioned above has enabled the completion of this investigation report to be carried out smoothly, and once again, I am very grateful for their assistance throughout this project.

\section{REFERENCES}

[1] Workfront. "Project Life Cycle", 2020. [Online] Available: https://www.workfront.com/projectmanagement/life-cycle [Accessed on: 4 September 2020]

[2] APM. "What is Project Management?", 2020. [Online] Available: https://www.apm.org.uk/resources/what-is-projectmanagement/ [Accessed on: 4 September 2020]

[3] Project Management Institute. "What is Project Management", 2020 [Online] Available: http://pmi.org/about/learn-about-pmi/what-is-projectmanagement [Accessed on: 4 September 2020]
[4] M. Symonds, "15 Causes of Project Failure", 13 Jun 2011. [Online] Available: https://www.projectsmart.co.uk/15-causes-of-projectfailure.php [Accessed on: 4 September 2020].

[5] MDN Web Docs. "HTML: Hypertext Markup Language", 2020. [Online] Available: https://developer.mozilla.org/en-

US/docs/Web/HTML [Accessed on: 10 September 2020]

[6] MDN Web Docs. "Learn to style HTML using CSS", 2020. [Online] Available: https://developer.mozilla.org/en-US/docs/Learn/CSS [Accessed on: 10 September 2020]

[7] MDN Web Docs. "JavaScript", 2020. [Online] Available: https://developer.mozilla.org/enUS/docs/Web/JavaScript [Accessed on: 10 September 2020]

[8] E. You, "Vue.js" , 2020. [Online] Available: https://vuejs.org/ [Accessed on: 10 September 2020]

[9] Vuetify. "Why you should be using Vuetify", 2020 [Online] Available: https://vuetifyjs.com/en/introduction/why-vuetify/ [Accessed on: 27 January 2021]

[10] Bhuvaneswary, N., Prabu, S., Karthikeyan, S., Kathirvel, R. and Saraswathi, T., 2021. Low Power Reversible Parallel and Serial Binary Adder/Subtractor. Further Advances in Internet of Things in Biomedical and Cyber Physical Systems, p.151.

[11] L. Tan, K. Yu, A. K. Bashir, X. Cheng, F. Ming, L. Zhao, X. Zhou, "Towards Real-time and Efficient Cardiovascular Monitoring for COVID-19 Patients by 5G-Enabled Wearable Medical Devices: A Deep Learning Approach", Neural Computing and Applications, 2021, https://doi.org/10.1007/s00521021-06219-9

[12] K. Yu, Z. Guo, Y. Shen, W. Wang, J. C. Lin, T. Sato, "Secure Artificial Intelligence of Things for Implicit Group Recommendations", IEEE Internet of Things Journal, 2021, doi: 10.1109/JIOT.2021.3079574.

[13] L. Zhen, Y. Zhang, K. Yu, N. Kumar, A. Barnawi and Y. Xie, "Early Collision Detection for Massive Random Access in Satellite-Based Internet of Things," IEEE Transactions on Vehicular Technology, vol. 70, no. 5, pp. 5184-5189, May 2021, doi: 10.1109/TVT.2021.3076015.

[14] L. Tan, N. Shi, K. Yu, M. Aloqaily, Y. Jararweh, “A Blockchain-Empowered Access Control Framework for Smart Devices in Green Internet of Things", ACM 
Transactions on Internet Technology, vol. 21, no. 3, pp. 1-20, 2021,https://doi.org/10.1145/3433542.

[15] K. Yu, L. Tan, L. Lin, X. Cheng, Z. Yi and T. Sato, "Deep-Learning-Empowered Breast Cancer Auxiliary Diagnosis for 5GB Remote E-Health," IEEE Wireless Communications, vol. 28, no. 3, pp. 54-61, June 2021, doi: 10.1109/MWC.001.2000374.

[16] S. Chen, L. Zhang, Y. Tang, C. Shen, R. Kumar, K. Yu, U. Tariq, and A. K. Bashir, "Indoor Temperature Monitoring Using Wireless Sensor Networks: A SMAC Application in Smart Cities", Sustainable Cities and Society, vol. 61, p. 102333, July 2020.

[17] Shivappriya, S.N., Priyadarsini, M., Stateczny, A., Puttamadappa, C. and Parameshachari, B.D., 2021. Cascade object detection and remote sensing object detection method based on trainable activation function. Remote Sensing, 13(2), p.200.

[18] Kiran, P., Parameshachari, B.D., Yashwanth, J. and Bharath, K.N., 2021. Offline Signature Recognition Using Image Processing Techniques and Back Propagation Neuron Network System. SN Computer Science, 2(3), pp.1-8.
[19] Kumar, T.M., Reddy, K.S., Rinaldi, S., Parameshachari, B.D. and Arunachalam, K., 2021. A Low Area High Speed FPGA Implementation of AES Architecture for Cryptography Application. Electronics, 10(16), p.2023.

[20] Pham, D.V., Nguyen, G.L., Nguyen, T.N., Pham, C.V. and Nguyen, A.V., 2020. Multi-topic misinformation blocking with budget constraint on online social networks. IEEE Access, 8, pp.78879-78889.

[21] Naeem, M.A., Nguyen, T.N., Ali, R., Cengiz, K., Meng, Y. and Khurshaid, T., 2021. Hybrid Cache Management in IoT-based Named Data Networking. IEEE Internet of Things Journal.

[22] Nguyen, T.N., Liu, B.H. and Wang, S.Y., 2019. On new approaches of maximum weighted target coverage and sensor connectivity: Hardness and approximation. IEEE Transactions on Network Science and Engineering, 7(3), pp.1736-1751.

[23] Kumar, M.K., Parameshachari, B.D., Prabu, S. and liberata Ullo, S., 2020, September. Comparative Analysis to Identify Efficient Technique for Interfacing BCI System. In IOP Conference Series: Materials Science and Engineering (Vol. 925, No. 1, p. 012062). IOP Publishing. 